\title{
Novel definition and algorithm for chaining fragments with proportional overlaps
}

\author{
Raluca Uricaru, Alban Mancheron, Eric Rivals \\ LIRMM, CNRS and Université de Montpellier 2, Montpellier, France, \\ \{uricaru, mancheron, rivals\}@lirmm.fr
}

\begin{abstract}
Chaining fragments is a crucial step in genome alignment. Existing chaining algorithms compute a maximum weighted chain with no overlaps allowed between adjacent fragments. In practice, using local alignments as fragments, instead of MEMs, generates frequent overlaps between fragments, due to combinatorial reasons and biological factors, i.e. variable tandem repeat structures that differ in number of copies between genomic sequences. In this paper, in order to raise this limitation, we formulate a novel definition of a chain, allowing overlaps proportional to the fragments lengths, and exhibit an efficient algorithm for computing such a maximum weighted chain. We tested our algorithm on a dataset composed of 694 genome couples and accounted for significant improvements in terms of coverage, while keeping the running times below reasonable limits.
\end{abstract}

\section{Introduction}

In biology, genome comparison is used for gene annotation, phylogenetic studies, and even vaccine design $[12,2,7]$. Many bioinformatics programs for whole genome comparison involve a fragment chaining step, which seeks to maximize the total length of the chained fragments (eg, [6]). Given the set of $n$ shared genomic intervals, i.e. fragments, the Maximum Weighted Chain problem (MWC) is solved in $O(n \log n)$ time by dynamic programming when overlaps between adjacent fragments are forbidden $[10,1]$. Alternatively, Felsner et al. showed that this problem is a special case of the Maximum Weighted Independent Set problem in a trapezoid graph, which they solve by a sweep line algorithm over an equivalent box order representation of the graph [5]. These algorithms $[1,5]$ can be extended to handle fixed length overlap between adjacent fragments, but this is not sufficient to deal with the large differences in fragment length obtained even with small bacterial genomes [14]. Moreover, with those definitions the weight does not account for overlaps. An $O(n \log n)$ time algorithm for the MWC with Fixed Length Overlap problem was designed and used for mapping spliced RNAs on a genome [13], but the fixed bound on overlaps remains a limitation. To raise this limitation, we formulate the MWC with Proportional Length Overlap problem (MWC-PLO) and exhibit the first chaining algorithms allowing for overlaps that are proportional to the fragment lengths, and whose chain weight function 
accounts for overlap. Following Felsner et al., we use the box representation of a trapezoid graph and adapt the sweep line paradigm to this problem.

Small overlaps are often due to equality over a few base pairs at fragment ends due to randomness, since the alphabet has only four letters. Biological structures like tandem repeats (TR) that vary in number of copy units generate overlaps that are large relatively to the fragments involved. To illustrate this case, let $u, v, w$ be words and assume the sequences of two genomes $G_{a}, G_{b}$ are $G_{a}=u v v w$ and $G_{b}=u v v v w$, i.e. contains a variable TR of motif $v$. Then, uvv generates a local alignment between $G_{a}$ and $G_{b}$, as well as vvw, but both fragments overlap over $v$ in both $G_{a}$ and $G_{b}$. As $v$ can be arbitrary large compared to $u$ or $w$, proportional overlaps are needed to accomodate such cases.

The paper is organised as follows. Section 2 presents the chaining problem without overlaps, Section 3 define it with proportional overlaps and set the dynamic programming setup and algorithm that solve it. In Section 4 we exhibit a sweep line algorithm for this question, proves its correctness and complexities, while we study its performances in Section 5, and conclude in Section 6 .

\section{Preliminaries}

Boxes are axis parallel hyper-rectangles in $\mathbb{R}^{k}$, where each genome is associated with one axis. For simplicity, we consider the two dimensional case where $k=2$, i.e. comparing two genomes. The length on a genome of the fragment associated with a box is the projection of that box on the corresponding axis.

Let $\alpha \in\{1,2\}$ index the axis, and for any point $x \in \mathbb{R}^{2}$ let $P_{\alpha}(x)$ denote its projection on axis $\alpha$. Let $I$ be an interval of $\mathbb{R}$ and $\mathcal{I}$ be a set of disjoint intervals of $\mathbb{R}$; we denote by $|I|$ the length of $I$ and by $|\mathcal{I}|$ the sum of the lengths of intervals in $\mathcal{I}$. Let $B$ be a box of $\mathbb{R}^{2}$. The upper, resp. lower, corner of $B$ is denoted by $u(B)$, resp. $l(B)$. By extension, the interval corresponding to the projection of $B$ on axis $\alpha$ is denoted $P_{\alpha}(B)$. Let $<$ denote the classical dominance order between points of $\mathbb{R}^{2}$.

Definition 1 (Overlap free box dominance order). Let $B_{x}, B_{y}$ be two boxes of $\mathbb{R}^{2}$. We say that $B_{y}$ dominates $B_{x}$, denoted $B_{x} \ll B_{y}$, if $l\left(B_{y}\right)$ dominates $u\left(B_{x}\right)$ in $\mathbb{R}^{2}$. If neither $B_{x}$ dominates $B_{y}$, nor $B_{y}$ dominates $B_{x}$, then $B_{x}$ and $B_{y}$ are incomparable.

Felsner et al. showed how to transform a trapezoid graph into a box order, i.e. a set of boxes equipped with the dominance order $\ll$ such that pairs of incomparable boxes are in one-to-one correspondance with trapezoid pairs linked by edges of the graph. Hence, the Maximum Weighted Independent Set problem in a trapezoid graph is equivalent to the MWC problem in the corresponding box order [5]. Given an order, recall that a chain is a set of mutually comparable elements, and a maximal element in a set is one with no other element dominating it. Each chain has exactly one maximal element. 


\section{A novel tolerance definition for the maximum weighted chain problem in a box order}

To formulate a MWC with Proportional Length Overlap problem (MWC-PLO) in our framework, we need to redefine the dominance order to accept overlaps that are proportional to the boxes' projection lengths, and to propose a weight function that truly measures the coverage on each genome. By coverage, it is generally meant the total length of the genomic intervals covered by the selected fragments [9]. This requires that the chain weight counts only once a subinterval covered by several overlapping fragments.

Let $r \in[0,1[$ represent the maximal allowed overlap ratio between any two boxes.

Definition 2 ( $r$ tolerant dominance order). Let $B_{u}$ and $B_{v}$ two boxes. $B_{v}$ dominates $B_{u}$ on axis $\alpha$ in this tolerant dominance order, denoted by $B_{u} \ll_{r, \alpha} B_{v}$, if and only if

$$
P_{\alpha}\left(u\left(B_{u}\right)\right)-P_{\alpha}\left(l\left(B_{v}\right)\right) \leq r \min \left(\left|P_{\alpha}\left(B_{u}\right)\right|,\left|P_{\alpha}\left(B_{v}\right)\right|\right) .
$$

Now, we denote by $B_{u} \ll_{r} B_{v}$ the fact that $B_{v}$ dominates $B_{u}$ if and only if for each $\alpha \in\{1,2\} B_{u} \ll_{r, \alpha} B_{v}$.

It can be easily shown that the dominance between boxes implies the dominance between their upper, resp. lower, corners. Moreover, this tolerant dominance order is transitive (Proof in Appendix).

Property 1. Let $B_{t}, B_{u}$ two boxes such that $B_{t} \ll_{r} B_{u}$. Then $l\left(B_{t}\right)<l\left(B_{u}\right)$ and $u\left(B_{t}\right)<u\left(B_{u}\right)$.

Property 2. The dominance order $\ll_{r}$ is transitive.

From Property 1, one deduces the following corollary, which will help to compute efficiently the weight of overlapping boxes in a chain.

Corollary 1. Let $B_{t}, B_{u}, B_{v}$ be three boxes such that $B_{t} \ll_{r} B_{u} \ll_{r} B_{v}$. Then: $\left(B_{t} \cap B_{v}\right) \subset\left(B_{u} \cap B_{v}\right)$.

We define the weight of a box as the sum of lengths of its projections on all axis, and the weight of a chain of boxes as the sum of the coverages on each axis.

Definition 3 (Weight of a box, of a chain). Let $B$ be a box and $\alpha \in[1,2]$. Its weight on axis $\alpha$ is $w_{\alpha}(B):=\left|P_{\alpha}(B)\right|$, and its weight is $w(B):=\sum_{\alpha=1}^{2} w_{\alpha}(B)$. Let $m \in \mathbb{N}$ and $C:=\left(B_{1} \ll_{r} \ldots \ll_{r} B_{m}\right)$ be a chain of $m$ boxes. The weight of $C$ on axis $\alpha$, denoted $W_{\alpha}(C)$, is

$$
W_{\alpha}(C):=\left|\bigcup_{i=1}^{m} P_{\alpha}\left(B_{i}\right)\right|
$$

while its weight is $W(C):=\sum_{\alpha=1}^{2} W_{\alpha}(C)$. 
Note also that the weight of a box only depends on the endpoints of its projection on each axis, and hence, can be computed in constant time. Clearly, it can be easily seen that

$$
\begin{aligned}
W_{\alpha}(C) & =w_{\alpha}\left(B_{m}\right)+\sum_{j=1}^{m-1}\left|P_{\alpha}\left(B_{j}\right) \backslash \bigcup_{l=j+1}^{m} P_{\alpha}\left(B_{l}\right)\right| \\
& =w_{\alpha}\left(B_{m}\right)+\sum_{j=1}^{m-1}\left|P_{\alpha}\left(B_{j}\right) \backslash P_{\alpha}\left(B_{j+1}\right)\right| \text { by Corollary } 1 .
\end{aligned}
$$

The following easy property will also prove useful.

Property 3. Let $B_{t}, B_{u}$ two boxes such that $B_{t} \ll_{r} B_{u}$. Then

- $B_{t} \cap B_{u}$ is an, eventually empty, axis parallel rectangle of $\mathbb{R}^{2}$, and

- for $\alpha \in[1,2],\left|P_{\alpha}\left(B_{t}\right) \backslash P_{\alpha}\left(B_{u}\right)\right|=\left|P_{\alpha}\left(B_{t}\right) \backslash P_{\alpha}\left(B_{t} \cap B_{u}\right)\right|=w_{\alpha}\left(B_{t}\right)-$ $w_{\alpha}\left(B_{t} \cap B_{u}\right)$.

Now, we can define the MWC-PLO problem. Let $n \in \mathbb{N}$, and $\mathcal{B}^{\prime}:=\left\{B_{2}, \ldots B_{n-1}\right\}$ be the set of input boxes. For convenience, we add two dummy boxes, $B_{1}, B_{n}$, such that for all $1<i<n$ : $B_{1} \ll_{r} B_{i} \ll_{r} B_{n}$. Additionally, we set $w\left(B_{1}\right)=$ $w\left(B_{n}\right):=0$. Now, the input consists in $\mathcal{B}:=\left\{B_{1}, \ldots, B_{n}\right\}$.

Definition 4 (MWC with Proportional Length Overlap). Let $r \in[0,1[, n \in \mathbb{N}$, and $\mathcal{B}:=\left\{B_{1}, \ldots, B_{n}\right\}$ a set of boxes. The MWC with Proportional Length Overlap problem is to find in $\mathcal{B}$, according to the dominance order $\ll_{r}$, the chain $C$ that ends in $B_{n}$ and whose weight $W(C)$ is maximal.

The notation of $r, \mathcal{B}$, and $W(C)$ are valid throughout the paper. For any $1 \leq i \leq$ $n$, let us denote by $\mathcal{C}_{i}$ the set of chains ending in $B_{i}$, and by $W\left(B_{i}\right)$ the weight of the maximal weighted chain in $\mathcal{C}_{i}$ (not to be confounded with $w\left(B_{i}\right)$ ). From now on, all the considered boxes belong to $\mathcal{B}$ unless otherwise specified.

\subsection{A dynamic programming framework}

Let us show that MWC-PLO can be solved by a dynamic programming algorithm. Equation 1 suggests a recurrence equation to compute $W\left(B_{i}\right)$, with $W\left(B_{1}\right)=0$ and for all $1<i \leq n$ :

$$
W\left(B_{i}\right)=\max _{B_{j}: B_{j} \ll_{r} B_{i}} W\left(B_{j}\right)+\sum_{\alpha=1}^{2}\left|P_{\alpha}\left(B_{i}\right) \backslash P_{\alpha}\left(B_{j}\right)\right| .
$$

Obviously, this implies that for all $1 \leq j<n$ the value of $W\left(B_{j}\right)$ will be reused for computing $W\left(B_{i}\right)$ for every box $B_{i}$ such that $B_{j} \ll_{r} B_{i}$. Thus, MWC-PLO consists of overlapping subproblems, which suits to the framework of dynamic programming [4, chap. 15]. However, it is correct to use Equation 2 only if our problem satisfies the condition of optimal substructures [4, chap. 15]. In Theorem 1, we show this is true (Proof in Appendix). 
Theorem 1 (Optimality of substructures). Let $m, i_{1}, \ldots, i_{m}$ be integers belonging to $[1, n]$, and let $D:=\left(B_{i_{1}}, \ldots, B_{i_{m}}\right)$ be an optimal weighted chain among the chains in $\mathcal{C}_{i_{m}}$. Thus, $D^{\prime}:=\left(B_{i_{1}}, \ldots, B_{i_{m-1}}\right)$ is an optimal weighted chain among those in $\mathcal{C}_{i_{m-1}}$.

The MWC with Proportional Length Overlap can thus be solved by a dynamic programming algorithm, which uses two $n$-element arrays: $W[$.$] and Pred[.]$ to store for all $1 \leq i \leq n$ resp. the values of $W\left(B_{i}\right)$ and the predecessor of $B_{i}$ in an optimal weighted chain ending in $B_{i}$. This algorithm takes $O\left(n^{2}\right)$ time and $O(n)$ space; in Section 4 we prove a more efficient algorithm for MWC-PLO.

Theorem 2. A dynamic programming algorithm (Algorithm DP) solves the MWC with Proportional Length Overlap problem in $O\left(n^{2}\right)$ time and $O(n)$ space.

\section{A sweep line algorithm for MWC with Proportional Length Overlap}

Here, we exhibit a sweep line algorithm for the MWC with Proportional Length Overlap problem (see Algorithm 1), prove it and study its complexity.

\subsection{Outline of the algorithm}

Following Felsner et al., we give a sweep line algorithm in which a vertical line sweeps the boxes in the plane by increasing $x$-coordinates of their corners, stopping at the lower left and upper right corners of each box. To avoid visiting all possible predecessors as in the $O\left(n^{2}\right)$ dynamic programming algorithm when computing the best chain ending in $B_{x}$, we maintain a set, $\mathcal{A}$, of active boxes that can compete for being the optimal predecessor in that chain. But as predecessors can overlap $B_{x}$, this computation involves several steps, meaning that $W\left[B_{x}\right]$ and $\operatorname{Pred}\left[B_{x}\right]$ can be updated several times before getting their final value; this differs from the previous algorithm.

Let $\mathcal{P}$ be an array containing the $2 n$ points corresponding to $l()$ and $u()$ corners of the $n$ boxes in $\mathcal{B}$. Points in $\mathcal{P}$ are ordered on their $x$-coordinates; among the points having identical $x$-coordinates, lower corners are placed before upper corners. For each point, we store to which box and to which corner it corresponds to. In Algorithm 1, the main loop sweeps the points of $\mathcal{P}$ and processes in a different manner lower (lines 8-11) and upper corners (lines 12-24). We say a box $B_{x}$ is open when the sweep line is located between $l\left(B_{x}\right)$ and $u\left(B_{x}\right)$ inclusive, closed when the line has passed $u\left(B_{x}\right)$, and future when it lies before $l\left(B_{x}\right)$. These states are exclusive of each other, and partition at each moment $\mathcal{B}$ in three disjoint sets (see Figure 1a). All open boxes at each point are kept in a set $\mathcal{O}$ (lines 9,13 ). The weight of a chain ending in, say $B_{i}$, and passing by a predecessor of $B_{i}, B_{x}$, can only be computed when $B_{x}$ is closed (when $W\left[B_{x}\right]$ has reached its final value). If $P_{1}\left(u\left(B_{x}\right)\right)<P_{1}\left(l\left(B_{i}\right)\right)$ then this can be done when stopping at $l\left(B_{i}\right)$ (lines 10-11), while if $B_{x}$ overlaps $B_{i}$ on $x$-axis, then this is 
done when stopping at $u\left(B_{x}\right)$, and in the same time for all open boxes having $B_{x}$ as predecessor (lines 14-18). These two cases partition the possible predecessors of $B_{i}$ according to the location of their upper corners in two areas $A_{b}\left(B_{i}\right)$ and $A_{o}\left(B_{i}\right)$ (cf. Figure 1b).

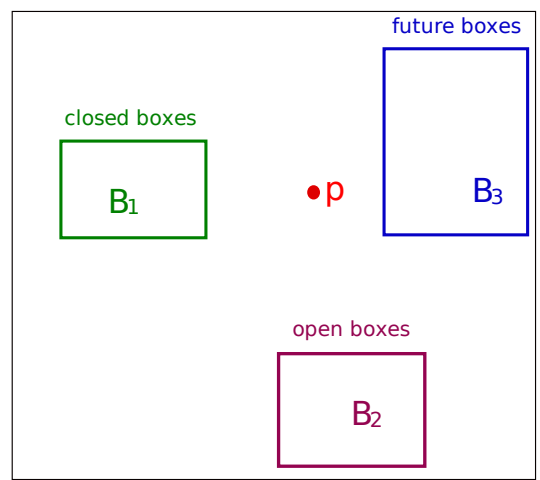

(a)

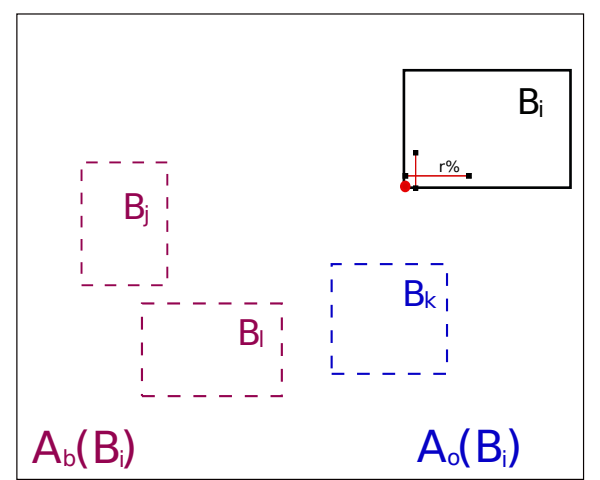

(b)

Fig. 1: (a) Example of boxes in each disjoint set forming a partition of $\mathcal{B}$, when sweeping a point $p$. (b) Partition of the search space of possible predecessors of $B_{i}$, according to the location of their upper corners, in two areas $A_{b}\left(B_{i}\right)$ and $A_{o}\left(B_{i}\right) . A_{b}\left(B_{i}\right)$ and $A_{o}\left(B_{i}\right)$ partition the rectangle delimited by a dashed line: $A_{b}\left(B_{i}\right)$ is at left from the line, and $A_{o}\left(B_{i}\right)$ at its right.

As above mentioned, we maintain in $\mathcal{A}$ the set of interesting predecessors for all future boxes. Boxes in $\mathcal{A}$ are active boxes. Hence, once closing a box (stopping at its upper corner), we test whether it should be turned active and inserted in $\mathcal{A}$ (lines 19-21). The current box, $B_{i}$, is inserted only if we cannot find a better predecessor in $\mathcal{A}$. Afterwards, if $B_{i}$ has been added, currently active boxes are investigated to determine if they are less interesting than $B_{i}$, in which case they are deleted from $\mathcal{A}$ (lines 22-24). Active boxes are consulted when opening a box $B_{i}$, for computing the best chain ending in $B_{i}$ with a predecessor in $A_{b}\left(B_{i}\right)$ (lines 10-11).

\subsection{Correctness of the Algorithm}

For $1 \leq i \leq n$, we show that $W\left[B_{i}\right]$ and $\operatorname{Pred}\left[B_{i}\right]$ stores the weight and the predecessor of $B_{i}$ in a maximum weighted chain ending in $B_{i}$. First, several simple invariants emerge from Algorithm 1. $I_{1}$ : At any point, the set $\mathcal{O}$ contains all open boxes. $I_{2}$ : Both $W\left[B_{i}\right]$ and $\operatorname{Pred}\left[B_{i}\right]$ they store their final values once $u\left(B_{i}\right)$ has been processed, since they are not altered after that point. $I_{3}$ : Hence, at any point all active boxes $($ i.e. boxes in $\mathcal{A}$ ), which are closed boxes, satisfy $I_{2}$. For conciseness, as $W\left[B_{i}\right]$ and $\operatorname{Pred}\left[B_{i}\right]$ are computed jointly, from now on we deal only with $W\left[B_{i}\right]$. Since potential predecessors of $B_{i}$ are partitioned in 


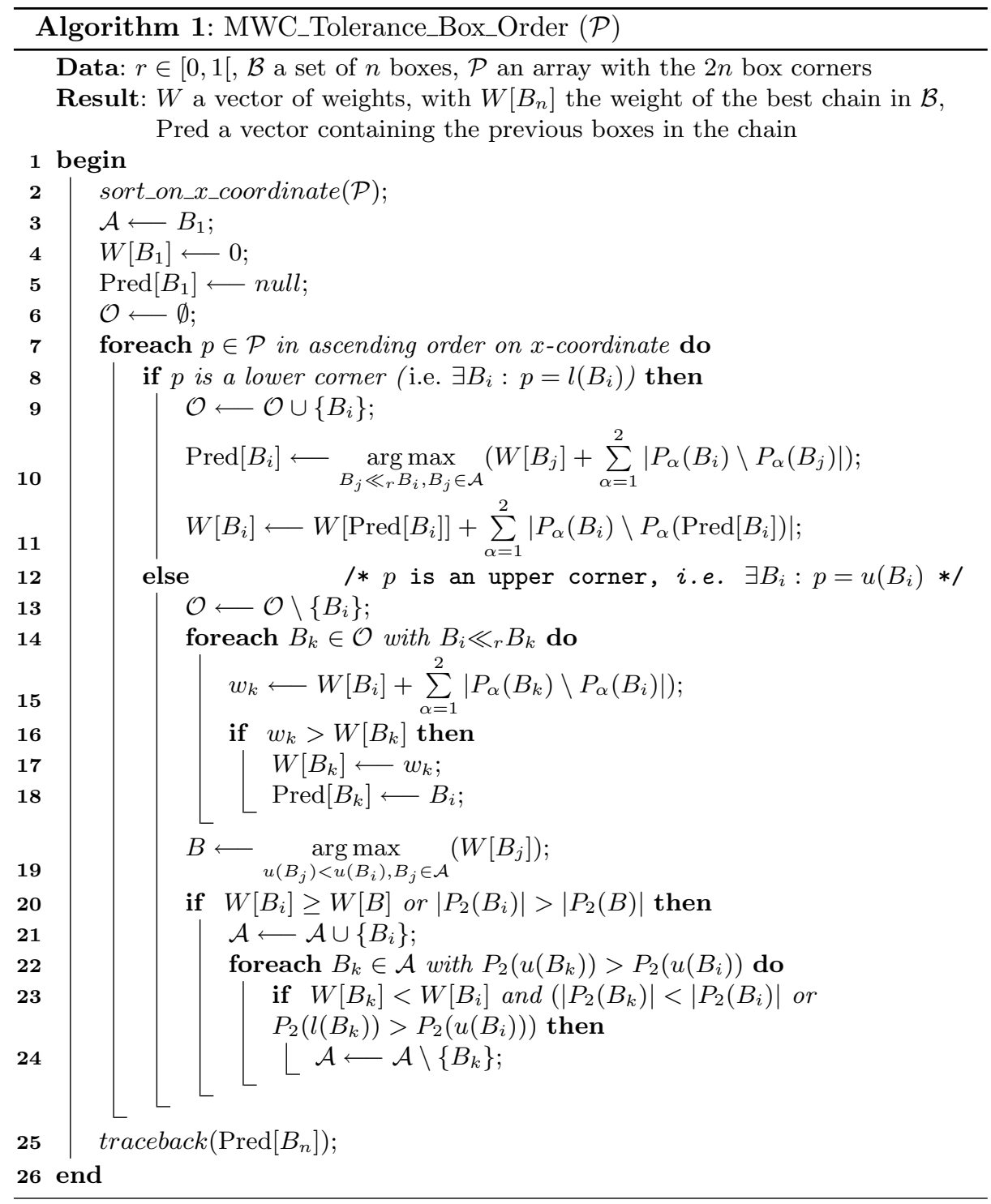


$A_{b}\left(B_{i}\right)$ (Figure 2a) and $A_{o}\left(B_{i}\right)$ (Figure $2 \mathrm{~b}$ ), we will prove two invariants: $I_{4}$ : partial optimality over $A_{b}\left(B_{i}\right)$ at lower corners, and $I_{5}$ : optimality at upper corners.

$I_{4}$ : partial optimality over $A_{b}\left(B_{i}\right)$ at lower corners We show that after processing $l\left(B_{i}\right), W\left[B_{i}\right]$ stores the weight of a maximum weighted chain ending in $B_{i}$ with predecessor in $A_{b}\left(B_{i}\right)$. Given line 10, this is equivalent to showing that no better chain ending in $B_{i}$ passes through a potential predecessor that does not belong to $\mathcal{A}$ at that point, which we prove by contradiction. While processing $l\left(B_{i}\right), \mathcal{A}$ contains a subset of boxes in $A_{b}\left(B_{i}\right)$, but obviously none from $A_{o}\left(B_{i}\right)$. Let $B$ be a closed box of $\mathcal{B} \backslash \mathcal{A}$ such that $B \ll_{r} B_{i}$ and $w\left(B_{i}\right)-w\left(B \cap B_{i}\right)+W[B]>W\left[B_{i}\right]$, in other words, $B$ makes a better predecessor for $B_{i}$ than those in $\mathcal{A}$. From $B \ll_{r} B_{i}$, we get

$$
P_{2}(u(B))-P_{2}\left(l\left(B_{i}\right)\right) \leq r \min \left(\left|P_{2}(B)\right|,\left|P_{2}\left(B_{i}\right)\right|\right) .
$$

As only two possibilities exist for $B$ not belonging to $\mathcal{A}$, we distinguish two exclusive cases.

$B$ was not turned active when sweeping $u(B)$ (lines 19-21). $B$ did not satisfy the condition on line 20. Let $B^{\prime}:=\underset{B_{j} \in \mathcal{A}: u\left(B_{j}\right)<u(B)}{\arg \max }\left(W\left[B_{j}\right]\right)$. Our hypothesis means that $u\left(B^{\prime}\right)<u(B)$ and

$$
\begin{gathered}
W[B]<W\left[B^{\prime}\right] \\
\left|P_{2}(B)\right| \leq\left|P_{2}\left(B^{\prime}\right)\right| .
\end{gathered}
$$

For $B$ does not overlap $B_{i}$ and $u\left(B^{\prime}\right)<u(B)$, we have $B^{\prime}$ does not overlap $B_{i}$ on the $x$-axis. From $u\left(B^{\prime}\right)<u(B)$, we get $P_{2}\left(u\left(B^{\prime}\right)\right)<P_{2}(u(B))$; this with equations 3 and 5 yields

$$
\begin{aligned}
P_{2}\left(u\left(B^{\prime}\right)\right)-P_{2}\left(l\left(B_{i}\right)\right) & <P_{2}(u(B))-P_{2}\left(l\left(B_{i}\right)\right) \\
& \leq r \min \left(\left|P_{2}(B)\right|,\left|P_{2}\left(B_{i}\right)\right|\right) \\
& \leq r \min \left(\left|P_{2}\left(B^{\prime}\right)\right|,\left|P_{2}\left(B_{i}\right)\right|\right) .
\end{aligned}
$$

Equation 6 and $B^{\prime}$ not overlapping $B_{i}$ on the $x$-axis imply $B^{\prime} \ll_{r} B_{i}$. Finally, from equations 4,5 , and $u\left(B^{\prime}\right)<u(B)$ we obtain:

$$
W[B]+\sum_{\alpha=1}^{2}\left(w_{\alpha}\left(B_{i}\right)-w_{\alpha}\left(B_{i} \cap B\right)\right)<W\left[B^{\prime}\right]+\sum_{\alpha=1}^{2}\left(w_{\alpha}\left(B_{i}\right)-w_{\alpha}\left(B_{i} \cap B^{\prime}\right)\right),
$$

and thus $B^{\prime}$ makes a better predecessor for $B_{i}$ than $B$, a contradiction.

$B$ was inactivated when sweeping $u\left(B_{k}\right)$ for some box $B_{k}$ ending before $l\left(B_{i}\right)$ (lines 22-24). The hypothesis means that $B$ was deleted from $\mathcal{A}$ for it satisfied $P_{2}\left(u\left(B_{k}\right)\right)<P_{2}(u(B)), W[B]<W\left[B_{k}\right]$, and at least one of the conditions (a) $\left|P_{2}(B)\right|<\left|P_{2}\left(B_{k}\right)\right|$ or (b) $P_{2}\left(u\left(B_{k}\right)\right)<P_{2}(l(B))$. 
a) As above (see Eq. 6), from Equation 4, from $\left|P_{2}(B)\right|<\left|P_{2}\left(B_{k}\right)\right|$, and $P_{2}\left(u\left(B_{k}\right)\right)<P_{2}(u(B))$, we get

$$
P_{2}\left(u\left(B_{k}\right)\right)-P_{2}\left(l\left(B_{i}\right)\right)<r \min \left(\left|P_{2}\left(B_{k}\right)\right|,\left|P_{2}\left(B_{i}\right)\right|\right) .
$$

Moreover, as $B_{k}$ does not overlap $B_{i}$ on $x$-axis, we obtain $B_{k} \ll_{r} B_{i}$. As $P_{2}\left(u\left(B_{k}\right)\right)<P_{2}(u(B)), B_{k}$ and $B$ do not overlap $B_{i}$ on $x$-axis, and $W[B]<$ $W\left[B_{k}\right]$, we finally derive

$$
\left.\left.W[B]+\sum_{\alpha=1}^{2}\left|P_{\alpha}\left(B_{i}\right) \backslash P_{\alpha}(B)\right|\right)<W\left[B_{k}\right]+\sum_{\alpha=1}^{2}\left|P_{\alpha}\left(B_{i}\right) \backslash P_{\alpha}\left(B_{k}\right)\right|\right) .
$$

b) By hypothesis, we know that $P_{2}\left(u\left(B_{k}\right)\right)<P_{2}(l(B))<P_{2}\left(l\left(B_{i}\right)\right)$, and since neither $B_{k}$ nor $B$ overlap $B_{i}$ on the $x$-axis, we directly obtain $B_{k} \ll B_{i}$ $\left(B_{i} \cap B_{k}=\emptyset\right)$. Thus, $W[B]<W\left[B_{k}\right]$ also implies Equation 8 .

With either condition (a) or (b), $B_{k}$ makes a better predecessor for $B_{i}$ than $B$, a contradiction.

Finally, after processing $l\left(B_{i}\right), W\left[B_{i}\right]$ stores the weight of a maximum weighted chain ending in $B_{i}$ with predecessor in $A_{b}\left(B_{i}\right)$, which concludes the proof of $I_{4}$.

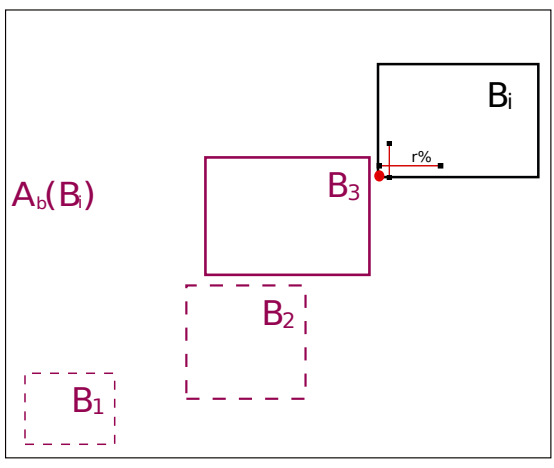

(a)

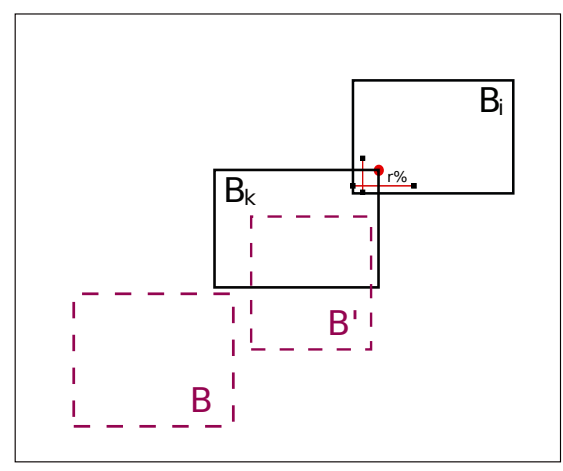

(b)

Fig. 2: (a) When the sweep line passes $l\left(B_{i}\right), \operatorname{Pred}\left[B_{i}\right]$ is a partial optimum on the set of possible predecessors of $B_{i}$ lying in $A_{b}\left(B_{i}\right)$. In the example, $B_{3}$ is the best current predecessor of $B_{i}$. (b) When the sweep line passes $u\left(B_{k}\right)$, Pred $\left[B_{i}\right]$ is a partial optimum on the set of possible predecessors of $B_{i}$ from $A_{b}\left(B_{i}\right) \cup\left\{B \in A_{o}\left(B_{i}\right) / P_{1}(u(B))<\right.$ $\left.P_{1}\left(u\left(B_{k}\right)\right)\right\}$.

$I_{5}$ : optimality at upper corners. We show that after processing $u\left(B_{i}\right), W\left[B_{i}\right]$ stores $W\left(B_{i}\right)$ (a mwc with a predecessor in $A_{b}\left(B_{i}\right) \cup A_{o}\left(B_{i}\right)$ ). As all predecessors of $B_{i}$ are closed, let us denote by $B$, the right most predecessor of $B_{i}$ on the $x$-axis: $B:=\arg \max _{B_{j} \ll_{r} B_{i}}\left(P_{1}\left(u\left(B_{j}\right)\right)\right)$. 
1. If $u(B) \in A_{b}\left(B_{i}\right)$ then all predecessors of $B_{i}$ are contained in $A_{b}\left(B_{i}\right)$. Hence, this situation was handled when processing $l\left(B_{i}\right)$, and Invariant $I_{4}$ regarding the partial optimality at lower corners, ensures that $W\left[B_{i}\right]$ stores $W\left(B_{i}\right)$.

2. If $u(B) \in A_{o}\left(B_{i}\right), W\left[B_{i}\right]$ has been correctly updated (lines 14-18), while $B_{i}$ was open, when sweeping $u\left(B_{k}\right)$ for each box $B_{k} \in \mathcal{B}$ such that $B_{k} \ll_{r} B_{i}$ and $u\left(B_{k}\right) \in A_{o}\left(B_{i}\right)$.

Hence, all predecessors of $B_{i}$ have been taken into account, and $W\left[B_{i}\right]$ stores $W\left(B_{i}\right)$. This concludes the proof of $I_{5}$, and closes the correctness proof.

\subsection{Time and space analysis}

Obviously, the sets $\mathcal{O}$ and $\mathcal{A}$ contain at most $n$ boxes, and thus require together with arrays Pred[.] and $W[],. O(n)$ space. We use balanced binary search trees (BST) to store $\mathcal{A}$ and $\mathcal{O}$, with boxes at the leaves ordered on $P_{2}(u()$.$) ,$ resp. $P_{1}(l()$.$) . Hence, the amortized time needed for all insertions, deletions,$ and rebalancing is $O(n \log n)$. However, looking for the active boxes that can be deleted at each execution of the outer loop (lines 22-24) may force us to examine all boxes in $\mathcal{A}$. As this is the more complex operation in the outer loop, we obtain an $O\left(n^{2}\right)$ worst case time complexity. Algorithm 1 maintains the subset of potential predecessors in $\mathcal{A}$ instead of searching through the whole box set as in Algorithm DP. The experimental running times observed when performing 694 whole genome comparisons show that, with this difference yields substantial improvements: Algorithm 1 takes seconds or, sometimes, minutes, where Algorithm DP, which is truly quadratic, takes minutes or hours, and even days, for values of $n$ ranging from 71 to $1,000,000$ fragments.

\section{Results}

An issue is whether allowing for overlaps improves the chain weight (here, the genome coverage) when comparing genomes, and at which computational cost. To investigate this issue, we compared the running times and coverages obtained without (using Chainer) and with proportional overlaps (using Algorithm 1) on 694 pairwise genome comparisons. Our comparison set consists in all pairwise genome comparisons of strains of the same bacteria (intra-species comparisons) as of Jan 2010: it comprises 346 different genomes from 87 species retrieved from Genome Reviews database [8]. First, we searched for local alignments between genome pairs using YASS with default parameters [11]. The output local alignments are the fragments given as input to the chaining step, for which we ran in parallel Chainer and Algorithm 1, with the weight of a fragment on each genome being the length of the aligned sequence. We authorized overlaps measuring up to $10 \%$ of the fragments' lengths $(r=0.1)$. Hence, the total chain weight, i.e. the sum of the chained fragments lengths minus the overlaps, computed by the chaining step gives the genome coverage, which we report as a percentage of the genome length. Of course as both chaining algorithms provide an optimal solution in their setup, the coverage with overlaps is larger than without overlaps. 


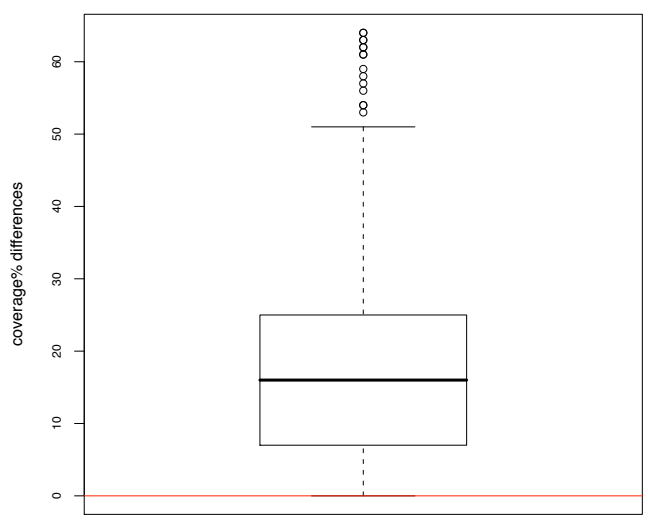

(a)

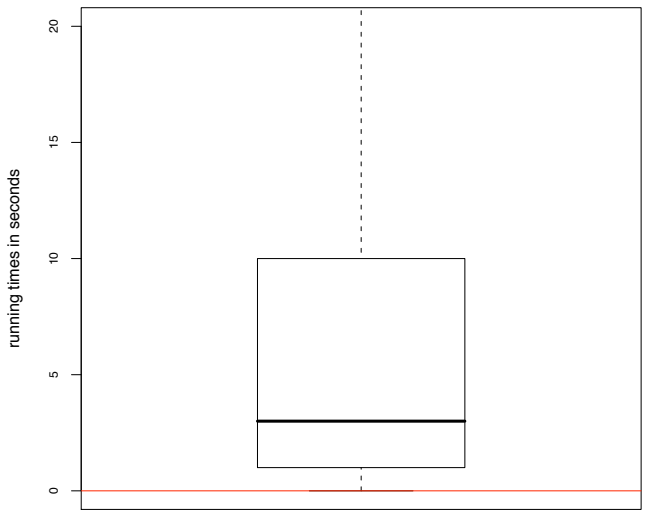

(b)

Fig. 3: (a) Differences in coverage obtained on bacterial genome comparisons between our algorithm and the classical chaining. The difference expresses which percentage of the genomes are additionally covered when allowing for overlaps. The results presented in a box-and-whiskers plot show the improvement in coverage brought by the acceptance of overlaps in the chain: in $50 \%$ of the cases it covers $15 \%$ more of the genomes. (b) Running times in seconds of our algorithm: in $75 \%$ of the cases the algorithm needs less than 10 seconds. 
Figure 3a plots the difference of coverages between both algorithms (e.g., a value of 10 meaning that chaining with overlaps covers $10 \%$ of the genome more than without overlaps). The box-and-whiskers plot shows that the improvement has a median of $15 \%$ and reaches values up to $60 \%$. Since bacterial genomes have a median length of $2.8 \mathrm{Mbp}$, a difference of one percent means at least 28 $\mathrm{Kbp}$ of additionally aligned sequences. Knowing that in average $87 \%$ of these genomes are coding and bacterial genes are $1 \mathrm{Kbp}$ long [15], 15\% more coverage will involve 420 additional genes compared to a solution without overlaps.

Chainer takes $<1 \mathrm{~s}$. in average and at most $17 \mathrm{~s}$. We plot the running times of Algorithm 1 in Figure 3b: below 10 seconds in $75 \%$ of the comparisons, and between 3 and 54 minutes in only 30 cases (those having up to one million input fragments). Thus, allowing for overlaps improves the coverage significantly at a reasonable cost.

A biological question regards the causes of such overlaps. For example, when comparing strains $C P 000046$ and $B A 000018$ of $S$. aureus the classical chaining results in a coverage of $65 \%$, where Algorithm 1 yields a $94 \%$ coverage. In fact, the chain obtained without allowing overlaps is interrupted by 17 holes of more than $10 \mathrm{kbp}$ each. For 14 of these holes, at least one large fragment (average size $37 \mathrm{kbp}$ ) was not included in the chain, because of an overlap with an adjacent fragment on one or both genomes. All overlaps measure between 1 bp and 1.8 $\mathrm{kbp}$ in length (average at $218 \mathrm{bp}$ ). This example shows that overlaps' lengths cannot be easily bounded by a constant. Large overlaps are due to variable tandem repeat structures that differ in number of copies between the strains. Correctly aligning such structures without breaking the region in two overlapping fragments requires a more general alignment model and specific algorithms [3].

\section{Conclusion}

To fulfil new needs in computational biology, we extended the classical framework of Maximum Weighted Chain by authorizing overlap between fragments in the computed chain, and formalized the Maximum Weighted Chain with Proportional Length Overlap problem where overlaps are proportional to the fragment lengths. Difficulties arise from the fact that the weights of overlaps are deduced from the chain weight. We exhibited the first two algorithms for this problem, which both solve it in quadratic time in function of the number of fragments. Experiments on real data sets show that i/ overlaps improve significantly the coverage of genomes (median of $15 \%$ ), ii/ our sweep line algorithm outperforms the truly quadratic dynamic programming solution in practice. However, the study of the average time complexity of the sweep line algorithm remains open. Comparing with fixed overlaps, as well investigating the robustness with respect to ratio of allowed overlaps are future lines of research.

Acknowledgements: RU beneficiates from a $\mathrm{PhD}$ fellowship from the French Ministery of Research. This work is supported by the ANR project CoCoGen (ANR-07-BLAN-0365). 


\section{References}

1. Mohamed Ibrahim Abouelhoda and Enno Ohlebusch. Chaining algorithms for multiple genome comparison. J. of Discrete Algorithms, 3:321-341, 2005.

2. Bastien Boussau and Vincent Daubin. Genomes as documents of evolutionary history. Trends in Ecology 83 Evolution, 25(4):224 - 232, 2010.

3. Sèverine Bérard and Eric Rivals. Comparison of minisatellites. J. of Computational Biology, 10(3-4):357-372, 2003.

4. Thomas H. Cormen, Charles E. Leiserson, Ronald L. Rivest, and Clifford Stein. Introduction to Algorithms. MIT Press, 2nd edition, 2001.

5. Stefan Felsner, Rudolf Muller, and Lorenz Wernisch. Trapezoid graphs and generalizations, geometry and algorithms. Discrete Applied Mathematics, 74:13-32, 1995.

6. Michael Hohl, Stefan Kurtz, and Enno Ohlebusch. Efficient multiple genome alignment. Bioinformatics, 18(S1):S312-320, 2002.

7. Andrew P. Jackson, John A. Gamble, Tim Yeomans, Gary P. Moran, David Saunders, David Harris, Martin Aslett, Jamie F. Barrell, Geraldine Butler, Francesco Citiulo, David C. Coleman, Piet W.J. de Groot, Tim J. Goodwin, Michael A. Quail, Jacqueline McQuillan, Carol A. Munro, Arnab Pain, Russell T. Poulter, MarieAdèle Rajandream, Hubert Renauld, Martin J. Spiering, Adrian Tivey, Neil A.R. Gow, Barclay Barrell, Derek J. Sullivan, and Matthew Berriman. Comparative genomics of the fungal pathogens Candida dubliniensis and Candida albicans. Genome Research, 19(12):2231-2244, 2009.

8. Paul Kersey, Lawrence Bower, Lorna Morris, Alan Horne, Robert Petryszak, Carola Kanz, Alexander Kanapin, Ujjwal Das, Karine Michoud, Isabelle Phan, Alexandre Gattiker, Tamara Kulikova, Nadeem Faruque, Karyn Duggan, Peter Mclaren, Britt Reimholz, Laurent Duret, Simon Penel, Ingmar Reuter, and Rolf Apweiler. Integr8 and Genome Reviews: integrated views of complete genomes and proteomes. $\mathrm{Nu}$ cleic Acids Research, 33(suppl_1):D297-302, 2005.

9. Claire Lemaitre and Marie-France Sagot. A small trip in the untranquil world of genomes. Theoretical Computer Science, 395:171-192, 2008.

10. Gene Myers and Webb Miller. Chaining multiple-alignment fragments in subquadratic time. In Proc. of the sixth annual ACM-SIAM symposium on Discrete algorithms (SODA), pages 38-47, 1995.

11. Laurent Noe and Gregory Kucherov. YASS: enhancing the sensitivity of DNA similarity search. Nucleic Acids Research, 33(S2):W540-543, 2005.

12. Davide Serruto and Rino Rappuoli. Post-genomic vaccine development. FEBS Letters, 580(12):2985 - 2992, 2006.

13. Tetsuo Shibuya and Igor Kurochkin. Match chaining algorithms for cDNA mapping. In Proc. Workshop on Algorithms in Bioinformatics (WABI), volume 2812 of Lecture Notes in Computer Science, pages 462-475. Springer, 2003.

14. Raluca Uricaru, Célia Michotey, Laurent Noé, Hélene Chiapello, and Eric Rivals. Improved sensitivity and reliability of anchor based genome alignment. In Irena Rusu et Eric Rivals, editor, Actes des Journées Ouvertes Biologie Informatique Mathématiques (JOBIM), pages 31-36, Nantes, 9-11th June 2009.

15. Lin Xu, Hong Chen, Xiaohua Hu, Rongmei Zhang, Ze Zhang, and Z. W. Luo. Average Gene Length Is Highly Conserved in Prokaryotes and Eukaryotes and Diverges Only Between the Two Kingdoms. Mol Biol Evol, 23(6):1107-1108, 2006. 


\section{A Additional proofs}

Proof (Proof of Property 2(transitivity of $\left.\ll_{r}\right)$ ). Let $B_{t}, B_{u}, B_{v}$ be three boxes such that $B_{t} \ll_{r} B_{u}$ and $B_{u} \ll_{r} B_{v}$. We will show that $B_{t} \ll_{r} B_{v}$. Let $\alpha \in\{1,2\}$. By hypothesis and from Property 1 , we obtain both $l\left(B_{t}\right)<l\left(B_{u}\right)<l\left(B_{v}\right)$ and $u\left(B_{t}\right)<u\left(B_{u}\right)<u\left(B_{v}\right)$. From these we get both

$$
P_{\alpha}\left(u\left(B_{t}\right)\right)-P_{\alpha}\left(l\left(B_{v}\right)\right)<P_{\alpha}\left(u\left(B_{t}\right)\right)-P_{\alpha}\left(l\left(B_{u}\right)\right) \leq r \min \left(\left|P_{\alpha}\left(B_{t}\right)\right|,\left|P_{\alpha}\left(B_{u}\right)\right|\right),
$$

and

$P_{\alpha}\left(u\left(B_{t}\right)\right)-P_{\alpha}\left(l\left(B_{v}\right)\right)<P_{\alpha}\left(u\left(B_{u}\right)\right)-P_{\alpha}\left(l\left(B_{v}\right)\right) \leq r \min \left(\left|P_{\alpha}\left(B_{u}\right)\right|,\left|P_{\alpha}\left(B_{v}\right)\right|\right)$.

When combined, these equations imply

$$
\begin{aligned}
P_{\alpha}\left(u\left(B_{t}\right)\right)-P_{\alpha}\left(l\left(B_{v}\right)\right) & \leq r \min \left(\left|P_{\alpha}\left(B_{t}\right)\right|,\left|P_{\alpha}\left(B_{u}\right)\right|,\left|P_{\alpha}\left(B_{v}\right)\right|\right) \\
& \left.\leq r \min \left(\left|P_{\alpha}\left(B_{t}\right)\right|,\left|P_{\alpha}\left(B_{v}\right)\right|\right)\right),
\end{aligned}
$$

and hence $B_{t} \ll_{r} B_{v}$.

Proof (Proof of Theorem 1 (Optimality of substructures)). By hypothesis, Equation 1 and Property 3, one has

$$
\begin{aligned}
W(D) & =W\left(B_{i_{m}}\right) \\
& =w\left(B_{i_{m}}\right)+\sum_{j=i_{1}}^{i_{m-1}} \sum_{\alpha}\left|P_{\alpha}\left(B_{j}\right) \backslash P_{\alpha}\left(B_{j+1}\right)\right| \\
& =w\left(B_{i_{m}}\right)-w\left(B_{i_{m}} \cap B_{i_{m-1}}\right)+w\left(B_{i_{m-1}}\right)+\sum_{j=i_{1}}^{i_{m-2}} \sum_{\alpha}\left|P_{\alpha}\left(B_{j}\right) \backslash P_{\alpha}\left(B_{j+1}\right)\right| \\
& =w\left(B_{i_{m}}\right)-w\left(B_{i_{m}} \cap B_{i_{m-1}}\right)+W\left(D^{\prime}\right) .
\end{aligned}
$$

We proceed by contradiction and assume that $E^{\prime}$, rather than $D^{\prime}$, is an optimal weighted chain ending in $B_{i_{m-1}}$, i.e. $W\left(E^{\prime}\right)>W\left(D^{\prime}\right)$. Consider the chain $E:=$ $D^{\prime} \cup\left\{B_{i_{m}}\right\}$. By the same reasoning as above, one has

$$
W(E)=w\left(B_{i_{m}}\right)-w\left(B_{i_{m}} \cap B_{i_{m-1}}\right)+W\left(E^{\prime}\right),
$$

and hence, $W(E)>W\left(B_{i_{m}}\right)$, contradicting the hypothesis that $D$ is an optimal weighted chain ending in $B_{i_{m}}$. MWC-PLO satisfies the condition of substructures' optimality. 